\title{
Exploring RNA Oligomerization and Ligand Binding by Fluorescence Correlation Spectroscopy and Small Angle X-Ray Scattering
}

\author{
Eileen Magbanua, Petr V. Konarev, Dmitri I. Svergun, \\ and Ulrich Hahn
}

\begin{abstract}
RNA forms defined structures and binds specifically to target molecules. The combination of data which results from fluorescence correlation spectroscopy (FCS) and small angle X-ray scattering (SAXS) measurements can be used to determine intermolecular interactions between RNA and its binding partners. To define oligomerization states of free RNA and its complexes with bound target molecules, hydrodynamic radii, radii of gyration as well as the maximum sizes of the components have to be determined and compared. Furthermore, the program OLIGOMER allows calculating the portions of monomeric and dimeric RNA, for instance, within a mixture.
\end{abstract}

Key words FCS, FCCS, SAXS, RNA dimerization, RNA oligomerization, Aptamers

\section{Introduction}

Aptamers are one group of nucleotides that bind target molecules with high affinity and specificity due to their three-dimensional structure. The target molecules can range from low molecular weight components to complex proteins. Depending on the binding mode between target and aptamer, the aptamer can interact as monomer or dimer for instance. This oligomerization state might change dependent on the bound molecule [1]. To monitor the dynamics of free and/or complexed aptamers one can use fluorescence correlation spectroscopy (FCS) and small angle X-ray scattering (SAXS).

FCS is a fluorescence-based method to study molecular interactions based on changes in mobility [2]. FCS requires fluorescence labeling of at least one of the interacting components. In case of two associated molecules the lower molecular weight component is fluorescently labeled and the complex has to differ 
significantly from the fluorescently labeled item regarding molar mass or rather diffusion time. To investigate the interaction between those two molecules, first the diffusion time of the fluorescently labeled component has to be characterized to calculate its hydrodynamic radius. Then the second unlabeled component is stepwise added yielding a significant increase of the diffusion time. The proportion of the different fluorescing components as well as their diffusion times can be calculated applying the two-component model. Using diffusion time of the complex, the hydrodynamic radius can be defined.

Fluorescence cross correlation spectroscopy (FCCS) is used to identify and characterize interactions between two fluorescing components [3]. The big advantage of FCCS over FCS is the fact that mass differences of the components investigated are irrelevant. Labeling the examined RNA with different fluorophores allows investigating its intermolecular dimerization.

SAXS is a powerful method for the analysis of biological macromolecules in solution [4]. Over the last decade, major advances in instrumentation and computational methods have led to new and exciting developments in the application of SAXS to structural biology including globular proteins, macromolecular complexes but also flexible systems [5-9]. SAXS can probe structure on an extremely broad range of macromolecular sizes, ranging from small proteins and polypeptides (a few $\mathrm{kDa}$ ), to macromolecular complexes and large viral particles (to several hundred MDa). RNA molecules can be measured in solution under near native conditions, and the effect of changes in sample environment (like $\mathrm{pH}$, temperature, ionic strength, ligand addition) can be easily followed. Still, the sample solutions must be well prepared, thoroughly purified and characterized before doing SAXS experiments. In such an experiment, samples containing dissolved macromolecules are exposed to an X-ray beam and the scattered intensity is recorded by a detector as a function of the scattering angle. Dilute aqueous solutions of nucleic acids give rise to an isotropic scattering intensity $I$, which depends on the modulus of the momentum transfer $s$ $(s=4 \pi \sin (\theta) / \lambda$, where $\lambda$ is the wavelength of the beam, and $2 \theta$ is the angle between the incident and scattered beam). The solvent scattering is subtracted and the background corrected intensity is presented as a radially averaged one-dimensional curve $I(s)$. For monodisperse solutions of identical randomly oriented molecule, the SAXS curve is proportional to the scattering of a single particle averaged over all orientations.

Several overall parameters can be directly obtained from an SAXS curve providing information about the size, oligomeric state, and overall shape of the molecule. Moreover, for folded nucleic acids or macromolecular complexes, low resolution threedimensional structures can be determined from the scattering data either $\mathrm{ab}$ initio or through the refinement using available high 
resolution structures and/or homology models [10]. SAXS is routinely employed for the validation of structural models, analysis of oligomeric states and the estimation of volume fractions of components in mixtures. SAXS has been actively used also for flexible systems including solutions of intrinsically disordered proteins (IDPs) [11]. In the present chapter the experimental and methodological peculiarities of the use of FCS and SAXS for exploring RNA oligomerization and ligand binding are presented.

\section{Materials}

Prepare all solutions RNase-free (see Note 1).

\subsection{FCS and FCCS}

1. ConforCor2 (Carl Zeiss) and software package.

2. Cover slip $24 \times 50 \mathrm{~mm}$.

3. Fluorophore or fluorescently labeled RNA (store at $-20^{\circ} \mathrm{C}$ ).

4. FCS buffer: $10 \mathrm{mM} \mathrm{Na-HEPES,} \mathrm{pH} \mathrm{7.4,} 100 \mathrm{mM} \mathrm{KCl,} 5 \mathrm{mM}$ $\mathrm{MgCl}_{2}$.

5. $100 \mathrm{mM} \mathrm{MgCl} 2$ stock solution.

6. For FCCS measurements: Fluorescently labeled RNA (see Note 2) with two different fluorophores, respectively (e.g., ATTO 488 and ATTO 647 N). Store at $-20{ }^{\circ} \mathrm{C}$.

7. RNase-free water.

\subsection{SAXS}

1. RNA solution: Precise knowledge of the RNA concentration is important for the assessment of the MW and oligomeric state of the RNA. The SAXS typical solute concentrations range from about $0.5-1 \mathrm{mg} / \mathrm{mL}$ to about $5-10 \mathrm{mg} / \mathrm{mL}$ (see Note 3 ), such that about $1-2 \mathrm{mg}$ of purified material is usually required for a complete SAXS experiment on the given construct at the given conditions (e.g., buffer composition, temperature). RNA is stored at $-20{ }^{\circ} \mathrm{C}$.

2. SAXS buffer: $20 \mathrm{mM}$ Na-HEPES, pH 7.4, $180 \mathrm{mM} \mathrm{KCl}$, $50 \mathrm{mM} \mathrm{MgCl}$, and $2 \mathrm{mM}$ dithiothreitol (DTT); stored at $-20{ }^{\circ} \mathrm{C}$. This buffer composition was used for SAXS experiments of fluorophore binding RNA aptamer in ref. [1]. Sufficient amount $(>10 \mathrm{~mL})$ of matching buffer(s) must be brought to the SAXS station to make the buffer collections and to dilute the samples if necessary. The buffer composition must precisely match the composition of the sample: even small mismatches in the chemical composition of the solvent between the buffer and the sample may lead to difficulties during background subtraction. At best, the last dialysis buffer should be used for the background measurements (see Note 4). 
2.3 SAXS Data Analysis
We present here the protocols based on the use of the program package ATSAS [12], which allows one to perform major processing and analysis steps. This package and all the programs mentioned below are publicly available for download from http://www.embl-hamburg.de/biosaxs/software.html.

\section{Methods}

\subsection{RNA}

3.2 RNA

Denaturation

and Renaturation

3.3 FCS

Measurement
1. Produce RNA by standard in vitro T7 transcription followed by gel-purification, extraction, and alcohol precipitation.

2. Alternatively, RNA can be chemically synthesized and purchased elsewhere.

3. For FCS titration experiments dilute RNA to a final concentration of $70 \mu \mathrm{M}$ in FCS buffer without $\mathrm{MgCl}_{2}$ (see Note 5).

4. For SAXS measurements prepare RNA in SAXS buffer with concentrations up to $45 \mu \mathrm{M}$.

1. Prior to the experiments heat the RNA to $70{ }^{\circ} \mathrm{C}$ for $10 \mathrm{~min}$ and transfer it directly on ice.

2. Add $\mathrm{MgCl}_{2}$ to a final concentration of $5 \mathrm{mM}$ to the RNA (see Note 6).

3. Incubate RNA for $\mathrm{l} h$ at room temperature to allow RNA to fold properly.

FCS titration experiments are carried out at room temperature $\left(22^{\circ} \mathrm{C}\right)$.

1. Adjust pinhole with a standard fluorophore in $x, y$, and $z$ direction.

2. Define the diffusion time $\tau_{\text {Diff; } i}$ of the fluorescent component.

3. Add non-fluorescent binding component to the fluorescent component and diffusion time increases significantly (see Note 7).

4. Titrate the non-fluorescent component stepwise to fluorescent component until diffusion time remains constant ( see Note 8).

5. The diffusion time of the complex can be determined easily by fitting FCS data with Zeiss software. Therefore, select the twocomponent model and set the diffusion time of the unbound fluorescent component as fixed value.

The two-component model describes free three-dimensional diffusion and triplet excited state with Eq. I

$$
G(\tau)=1+\frac{1}{N} \cdot\left(\frac{1-T+T \cdot \mathrm{e}^{-\frac{\tau}{\tau_{T}}}}{1-T}\right) \cdot\left\{\sum_{i=1}^{2} \frac{f_{1}}{\left(1+\frac{\tau}{\tau_{D i f f ; i}}\right) \sqrt{1+\frac{\tau}{\tau_{D i f f ; i}} S^{-2}}}\right\}
$$


and uses number of particles $N$, diffusion time $\tau_{\text {Diff, } i}$, fractional population $f_{1}$ of two different diffusion species $(n=2)$ with triplet fraction $T$, lag time $\tau$, and structure parameter $S$.

6. Calculate the complex fraction $\Upsilon$ using the concentration of complex $[C]$ and free fluorescent component $[F]$ :

$$
\Upsilon=\frac{[C]}{[F]+[C]} .
$$

7. To calculate the dissociation constant $K_{\mathrm{d}}$ plot the complex fraction $\Upsilon$ against concentration of non-fluorescent species. $K_{\mathrm{d}}$ equates to the non-fluorescent species concentration at the half-maximum of complex fraction $\Upsilon$.

8. Use diffusion time $\tau_{\text {Diff } ;}$ to calculate diffusion coefficient $D_{i}$ by using radial diameter $r_{0}$ of standard fluorophores (Alexa $488=169 \mathrm{~nm}, \mathrm{R} 6 \mathrm{G}=189 \mathrm{~nm}$, and $\mathrm{Cy} 5=250 \mathrm{~nm})$ and

$$
D_{i}=\frac{r_{0}^{2}}{4 \tau_{D i f f ; i}} .
$$

9. Calculate the hydrodynamic radius $r_{H}$ by

$$
r_{H}=\frac{\kappa T}{\left(6 \pi \eta D_{i}\right)}
$$

and applying the Boltzmann constant $\kappa\left(138 \times 10^{-23} \mathrm{~kg} \mathrm{~m}^{2} \mathrm{~s}^{-2} \mathrm{~K}^{-1}\right)$, the viscosity $\eta\left(1.002 \times 10^{-3}\right.$ $\left.\mathrm{kg} \mathrm{m}^{-1} \mathrm{~s}^{-1}\right)$, temperature $T\left(22{ }^{\circ} \mathrm{C}=295.15 \mathrm{~K}\right)$, and calculated diffusion coefficient $D_{i}$.

3.4 SAXS Measurement

3.4.1 General

Preparative Steps
A typical sample volume required for a single measurement is about 20-60 $\mu \mathrm{L}$ depending on the SAXS station used (see Note 9). The measurements of the SAXS data are usually made below room temperature (typically, at $5-10{ }^{\circ} \mathrm{C}$ ) to reduce the possible radiation damage effects ( see Note 10).

1. Check for radiation damages during X-ray measurements. If the effect is observed, measures should be taken (reduction of the data takes time, flow of the sample during exposure, addition of glycerol or DTT as scavengers).

2. Record a series of scattering curves at varying concentrations to ensure that the conditions of a 'dilute' solution are fulfilled. For fluorophore binding RNA aptamer [1] concentrations ranging from 0.2 to $0.8 \mathrm{mg} / \mathrm{mL}$ were used.

3. Measure corresponding buffer before and after the sample (see Notes 11 and 12). 
3.4.2 The SAXS

Experiment

\subsection{SAXS Data Analysis}

3.5.1 Primary Analysis of SAXS Data
1. Perform SAXS measurements of RNA alone and of the corresponding buffer.

2. Add binding component in excess to RNA to ensure that all RNA molecules are bound.

3. Perform SAXS measurements of RNA in complex and of the corresponding buffer.

The scattering data are usually collected by two-dimensional detectors, and the images are appropriately processed, corrected, and reduced to one-dimensional scattering profiles by the local software of the SAXS stations. The output is typically an ASCII file containing the subtracted data in columnar format: $s, I(s), \sigma(s)$, where the latter column represents the standard deviation of the processed intensity. The normalization and buffer subtraction are performed with following Eq. 5

$$
I(s)=\frac{\left(\frac{I_{\text {sample }}(s)}{T_{\text {sample }}}-\frac{I_{\text {buffer }}(s)}{T_{\text {buffer }}}\right)}{c}
$$

where $I_{\text {sample }}(s)$ and $I_{\text {buffer }}(s)$, respectively, are the measured scattering data from the sample and the buffer, $T_{\text {sample }}$ and $T_{\text {buffer }}$ are the intensities of the transmitted X-ray beam, and $c$ is the solute concentration. This basic operation is often performed by the stationspecific software, scripts or completely automatically (the latter is the case, e.g., at the synchrotron P12 station of the EMBL in Hamburg). In the following we assume that the radially averaged and subtracted patterns are available and describe the sequence of actions using the interactive data analysis program PRIMUS from the ATSAS package [12].

1. Load the SAXS curve in PRIMUS.

2. Do a Kratky representation of the SAXS curve, which can be obtained with the SASPLOT application of PRIMUS that allows several data representations.

A Kratky representation of the SAXS curve $\left(I(s) \times s^{2}\right.$ vs $\left.s\right)$ is an excellent tool to qualitatively identify conformational disorder in biomacromolecules. Unstructured RNA presents a continuous raise of $I(s) \times s^{2}$, whereas globular constructs display a peak. Partly disordered systems present a mixed behavior depending on the relative proportion of each of the parts. An example of Kratky plots for the folded tRNA (PDB code: 4TRA.pdb), unfolded $\operatorname{DsrA}_{34}$ [13] and folded fluorophorebinding RNA aptamer [6] is given in Fig. 1.

3. Use Guinier approximation, $R_{g}$ and the forward scattering, $I(0)$ that derived from the initial part of the scattering profile [14]. At very small angles the intensity is represented as: 


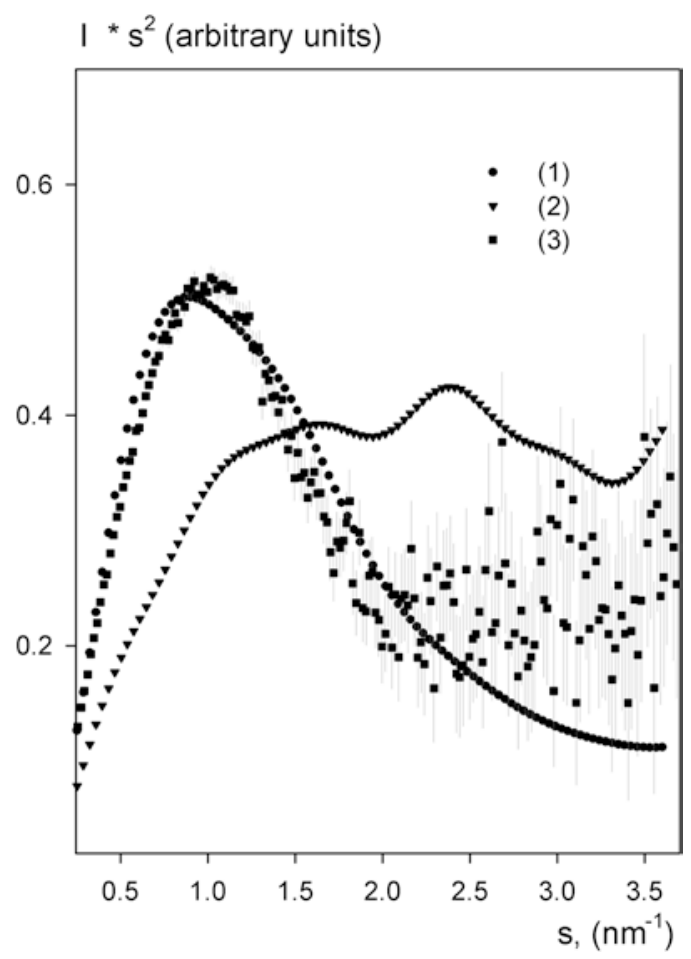

Fig. 1 SAXS Kratky plots of a folded tRNA (curve 1, circles), unfolded DsrA $A_{34}$ (curve 2, triangles), and folded SRB2m (curve 3, squares). The plot displays the weighted scattering intensity $\left(I \times s^{2}\right)$ as a function of momentum transfer $s=4 \pi \sin (\theta) / \lambda$, where $2 \theta$ is the scattering angle and $\lambda=0.15 \mathrm{~nm}$ is the X-ray wavelength. The theoretical scattering from tRNA and $\mathrm{DsrA}_{34}$ models are computed with CRYSOL

$$
I(s)=I(0) \exp \left(\frac{-\left(s R_{g}\right)^{2}}{3}\right)
$$

The $R_{g}$ and $I(0)$ are obtained by a simple linear fit in logarithmic scale using the Guinier option of PRIMUS where the optimal range of points for the analysis can be selected (see Note 13). Note that there is an option Autorg available, which selects the appropriate range and computes the $R_{g}$ automatically. In some cases, however, it might be useful to cross-validate the results by interactive analysis.

4. Divide $I(0)$ values by the concentration of the sample in $\mathrm{mg} / \mathrm{mL}$ to apply the equation.

$$
M W_{R N A}=\frac{I(0)_{R N A} \cdot M W_{\text {stand }}}{I(0)_{\text {stand }}}
$$


3.5.2 Ab Initio Shape Determination
5. Compare $I(0)$ value of the molecule studied with that obtained for a standard RNA molecule of known molecular weight (MW) and concentration (see Note 14). The forward intensity $I(0)$ provides the estimate of the $\mathrm{MW}$ of the molecule and hence suggests its oligomeric state.

6. Obtain the distance distribution function $P(r)$ from the SAXS curve with the program GNOM [15] which is called directly from PRIMUS or its automated version AUTOGNOM [16]. $P(r)$ is defined as the distribution of distances between volume elements insight the particle. $P(r)$ defines the maximum particle distance, $D_{\max }$ beyond which $P(r)$ is equal to zero. Porod's law [17] defines the asymptotic behavior of $I(s)$ for homogeneous particles at large angles which decays as $s^{4}$. Using the Porod invariant, the values of the excluded particle volume can be obtained from the scattering data using the Porod option of PRIMUS where the optimal range of points for the analysis can be selected ( see Note 15).

Distance distribution within the particle depends on its shape; therefore the scattering intensity can also be considered a shape's footprint. It is clear, however, that the reconstruction of a threedimensional model of an object from its one-dimensional scattering pattern is an ill-posed problem, as multiple structures can provide one and the same scattering profile. A simplification reducing the ambiguity of such reconstruction consists in representing the particle at low resolution as a homogeneous body. The model could be a complex shape built from densely packed beads on a regular grid. In a generic modeling approach the search of an optimal model is performed to minimize discrepancy between the experimental data $I_{\exp }(s)$ and a scattering profile computed from the model $I_{\text {calc }}(s)$ :

$$
\chi^{2}=\frac{1}{N-1} \sum_{N}^{j=1}\left[\frac{I_{\exp }\left(s_{j}\right)-c I_{c a l c}\left(s_{j}\right)}{\sigma\left(s_{j}\right)}\right]^{2},
$$

where $c$ is a scaling factor, $N$ is the number of points, and $\sigma$ denotes experimental errors. In practice, the target function to be minimized $\mathrm{TF}=\chi^{2}+\sum \alpha_{i} P_{i}$ contains not only the discrepancy term $\chi^{2}$ but also a set of weighted penalties $\alpha_{i} P_{i}$. The most wide-spread low resolution shape determination approach (implemented in programs DAMMIN/DAMMIF) employs simulated annealing minimization to build a compact interconnected ensemble of beads within a search volume (typically a sphere with the diameter $D_{\max }$ ) that fits the experimental data $[18,19]$. The natural restraints in this case are requirement of interconnectivity (a molecule cannot 
consist of separate fragments) and compactness (modeling is done at low resolution using homogeneous approximation; no fine details of the structure are expected).

The following steps should be made for ab initio shape determination analysis:

1. Run DAMMIN/DAMMIF for RNA molecule alone and with a ligand. Provide GNOM files as input data.

2. The output pdb files will contain ab initio shapes of the RNA molecule. Check the fit quality $\chi$ obtained with DAMMIN/DAMMIF, it should be around unity and the * fir file should not display systematic deviation between the experimental and calculated data. An example of ab initio model of SRB2 $\mathrm{m}$ with PBV (patent blue $\mathrm{V})$ computed by DAMMIN [6] is presented in Fig. 2.

3. Compare the volumes of the ab initio models for the RNA molecule alone and with a ligand in order to check whether the oligomerization state of RNA was changed (see Note 16).

3.6 Identification of Oligomerization State
In case of a polydisperse system without interparticle interactions, the scattering profile is a linear combination of the scattering
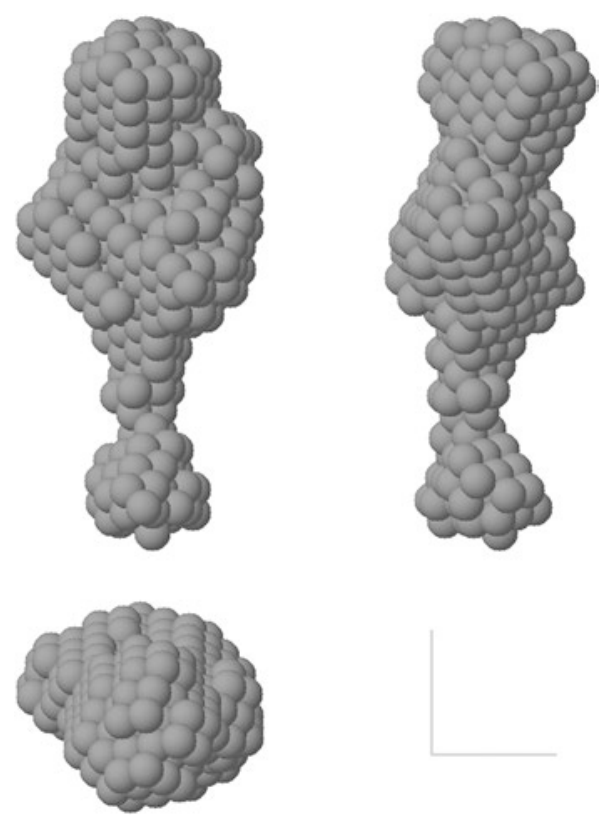

Fig. 2 The typical ab initio monomer model of SRB2m with PBV restored by DAMMIN. The right and bottom panels show the models rotated by 90 around the $y$ and $x$ axes, respectively. The scale bar is equal to $2 \mathrm{~nm}$. Reprinted with permission from: Werner, A., Konarev, P.V., Svergun, D.I., and Hahn, U. (2009) Characterization of a fluorophore binding RNA aptamer by fluorescence correlation spectroscopy and small angle X-ray scattering. Anal Biochem 389, 52-62 (Elsevier) 
intensities of individual components, weighted by their volume fractions [6] and is described by Eq. 9.

$$
I(s)=\frac{1}{N} \sum_{N}^{n=1} I_{n}(s)
$$

If the scattering patterns of all the mixture components are known (e.g., a mixture of monomers and dimers with known structure), the volume fractions are directly computed from SAXS data using the program OLIGOMER [20]. This approach is widely used to study oligomeric equilibria, complex formation, and structural transitions [21].

To identify the oligomerization state of the RNA the following steps should be performed:

1. The excluded volume in $\mathrm{nm}^{3}$ of RNA calculated by SAXS data is numerically equal to the molecular weight in $\mathrm{kDa}$ [22]. Compare $V_{p}$ and MW of RNA. If values differ this provides a first indication for an oligomerization state.

2. Compare $V_{p}, D_{\max }, R_{g}$ (from SAXS data), and $r_{H}$ (from FCS data) RNA alone and in complex with bound component (see Table 1 with overall parameters obtained for fluorophorebinding RNA aptamer in ref. [6]). The bound component can influence oligomerization state of RNA, e.g., either to form monomers or dimers.

\section{Table 1}

Overall structural parameters of SRB2m RNA

\begin{tabular}{llllll}
\hline Sample & Method & $\mathrm{r}_{H}$ or $\boldsymbol{R}_{\boldsymbol{g}}(\mathbf{n m})$ & $\boldsymbol{D}_{\max }(\mathbf{n m})$ & $\boldsymbol{V}_{\boldsymbol{p}}\left(\mathbf{n m}^{3}\right)$ & oligom. state \\
\hline SRB2m & DLS & $3.1 \pm 0.4$ & & & \\
& SAXS & $2.8 \pm 0.1$ & $10.0 \pm 1.0$ & $38.0 \pm 4.0$ & ++ and + \\
\hline SRB2m + Sulforhodamine B & FCS & $2.9 \pm 0.1$ & & & \\
& SAXS & $2.5 \pm 0.1$ & $8.5 \pm 1.0$ & $30.5 \pm 3.0$ & ++ and + \\
\hline SRB2m + PBV & FCS & $1.9 \pm 0.1$ & & & \\
& SAXS & $2.3 \pm 0.1$ & $8.0 \pm 1.0$ & $23.5 \pm 3.0$ & + \\
\hline
\end{tabular}

Hydrodynamic radii $r_{H}$ determined by FCS or DLS and radii of gyration $R_{g}$ determined by SAXS, maximum sizes $D_{\text {max }}$ excluded volumes $V_{p}$ from SAXS studies of SRB2m, SRB2m + Sulforhodamine B and SRB2m+PBV (patent blue V). The oligomerization state is presented by ++ in case of dimeric molecules and by + in the case of monomeric molecules. The errors represent standard deviations. The data were derived from at least two independent experiments. Reprinted with permission from: Werner, A., Konarev, P.V., Svergun, D.I., and Hahn, U. (2009) Characterization of a fluorophore binding RNA aptamer by fluorescence correlation spectroscopy and small angle X-ray scattering. Anal Biochem 389, 52-62 (Elsevier) 
3. In case of oligomerization use the program OLIGOMER to calculate proportion of respective monomer and dimer, for instance. For calculation the results of SAXS measurements are required as well as molecular weight of RNA.

3.7 FCCS

Measurement
FCCS can also be used to identify oligomerization state of RNA molecules. Therefore the same RNA has to be fluorescently labeled with different fluorophores (see Note 17).

1. Mix both fluorescently labeled RNAs in equal amounts.

2. Denature and refold RNA following steps $\mathbf{1 - 3}$ in Subheading 3.2.

3. Measure the cross-correlation signal. Cross-correlation signal should occur in case of oligomerization of RNA.

1. Aqueous solutions can be treated with diethylpyrocarbonate (DEPC) to inactivate RNases due to acetylation of histidin, lysine, cysteine, and tyrosine residues. Therefore solution is treated with $0.1 \%(\mathrm{v} / \mathrm{v})$ DEPC and stirred overnight. Autoclaving afterwards leads to destruction of DEPC. Trisbuffered solutions should not be treated with DEPC directly because it inactivates DEPC.

2. In case of precipitants or inhomogeneity RNA solution can be centrifuged for $2 \mathrm{~h}$ at maximum speed.

3. Typically, for each sample a concentration series (e.g., 1, 2, 5, $10 \mathrm{mg} / \mathrm{mL}$ ) has to be measured. If the sample tends to (or may) aggregate at higher concentrations, it is highly recommended to bring the low concentration stocks to the SAXS instrument and concentrate the samples prior to the X-ray measurements. If the sample is well-behaved, one may bring high concentration stocks to dilute them before the measurements.

4. To achieve the best SAXS results, the RNA molecules should normally be dissolved in the buffers yielding close to ideal solutions. The ionic strength and $\mathrm{pH}$ should be selected in such a way that the RNA molecules minimally interact with each other unless it is required by the nature of the experiment. Any additives (salts, small molecules, co-solutes, etc.) diminish the contrast of the RNA molecule and increase the $\mathrm{X}$-ray absorption; therefore buffers with excessive amounts of additives (typically, not more than $0.5 \mathrm{M} \mathrm{NaCl}$, not more than $5 \%$ glycerol, not more than $5 \mathrm{mM}$ ATP, etc.) should be avoided unless this is not dictated by the biological or chemical considerations. 
5. Concentration of RNA refers to titration experiments in which RNA binds to a fluorescent component. If RNA itself is fluorescently labeled and the interacting component is nonfluorescent, lower concentrations of RNA are used.

6. Heating RNA in presence of $\mathrm{MgCl}_{2}$ might lead to hydrolysis of RNA.

7. Molar mass of a molecule determines diffusion time with $\tau_{\text {Diff }} \approx \sqrt[3]{m}$. Therefore the molar masses of investigated molecules have to differ by a factor of 8 or higher for distinguishing between different diffusion species.

8. Volume differentials due to evaporation can be adjusted by adding RNase-free water.

9. On second generation synchrotrons and on older X-ray laboratory sources, one needs about $50 \mu \mathrm{L}$ and more of the sample; on modern high brilliance synchrotrons and on new laboratory cameras, about $10 \mu \mathrm{L}$ of the sample are required.

10. SAXS stations normally allow for controlled measurement of the temperature series, in the range from ice melting to water boiling points. The temperature measurements are often useful for the studies of the thermodynamic folding/unfolding processes.

11. Note that typically all the SAXS measurements for the given experimental session are performed in one and the same measuring cell to ensure that the background scattering remains unchanged allowing for reliable buffer subtraction. The sample compartment (which is either a cell with flat windows or a capillary) is cleaned and refilled after each measurement. In the past, the cleaning and filling procedure was done manually; nowadays, liquid handling robots are becoming more and more popular to facilitate the automatic data collection [23]. On some stations (e.g., a synchrotron P12 station of the EMBL in Hamburg) remote operation is possible where the users send the samples and perform the data collection from their laboratory.

12. On high brilliance synchrotrons, the samples can be damaged by the X-ray radiation even during the seconds and sub-seconds collection time. A reducing agent (e.g., 2 mM DTT or TCEP) or an additive slowing down the formation of free radicals (e.g., up to $5 \%$ glycerol) may be added to the sample before the experiment to diminish the damage/aggregation during the data collection. In any case, synchrotron data should be collected in short individual time frames, which have to be compared to each other to detect the radiation damage effects.

13. The momentum transfer range used for Guinier's approach must not exceed $s<1.3 \times R_{g}$. The first points of the curve, if 
they are influenced by the primary beam and show nonlinear behavior, must be suppressed from Guinier's analysis.

14. If one uses a standard protein molecule in Eq. 7, the experimental molecular weight of the RNA molecule from Eq. 7 will be two times overestimated due to the two times higher density of the RNA molecule compared to the protein.

15. There is a program AUTOPOROD available, which selects the appropriate range and computes the $V_{p}$ automatically. In some cases, however, it might be useful to cross-validate the results by interactive analysis.

16. Ab initio shape determination can be applied only for wellbehaved monodisperse systems.

17. A fluorophore can be inserted into RNA at the $5^{\prime}$ or $3^{\prime}$ end as well as intramolecularly. Location of the label is dependent on RNA binding site and should not interfere with binding component.

\section{References}

1. Werner A, Konarev PV, Svergun DI et al (2009) Characterization of a fluorophore binding RNA aptamer by fluorescence correlation spectroscopy and small angle X-ray scattering. Anal Biochem 389:52-62

2. Haustein E, Schwille P (2007) Fluorescence correlation spectroscopy: novel variations of an established technique. Annu Rev Biophys Biomol Struct 36:151-169

3. Weisshart K, Jungel V, Briddon SJ (2004) The LSM 510 META - ConfoCor 2 system: an integrated imaging and spectroscopic platform for single-molecule detection. Curr Pharm Biotechnol 5:135-154

4. Feigin LA, Svergun DI (1989) Structure analysis by small-angle $\mathrm{X}$-ray and neutron scattering. In: Taylor GW (ed) Acta polymerica, vol 40. Plenum Press, New York/London, p 224

5. Svergun DI, Koch MH (2002) Advances in structure analysis using small-angle scattering in solution. Curr Opin Struct Biol 12:654-660

6. Koch MH, Vachette P, Svergun DI (2003) Small-angle scattering: a view on the properties, structures and structural changes of biological macromolecules in solution. Q Rev Biophys 36:147-227

7. Petoukhov MV, Svergun DI (2005) Global rigid body modeling of macromolecular complexes against small-angle scattering data. Biophys J 89:1237-1250
8. Petoukhov MV, Svergun DI (2007) Analysis of X-ray and neutron scattering from biomacromolecular solutions. Curr Opin Struct Biol 17:562-571

9. Jacques DA, Trewhella J (2010) Small-angle scattering for structural biology-expanding the frontier while avoiding the pitfalls. Protein Sci 19:642-657

10. Mertens HD, Svergun DI (2010) Structural characterization of proteins and complexes using small-angle $\mathrm{X}$-ray solution scattering. J Struct Biol 172:128-141

11. Bernado P, Mylonas E, Petoukhov MV et al (2007) Structural characterization of flexible proteins using small-angle X-ray scattering. J Am Chem Soc 129:5656-5664

12. Konarev PV, Petoukhov MV, Volkov VV et al (2006) ATSAS 2.1, a program package for small-angle scattering data analysis. J Appl Cryst 39:277-286

13. de Almeida Ribeiro E Jr, Beich-Frandsen M, Konarev PV et al (2012) Structural flexibility of RNA as molecular basis for Hfq chaperone function. Nucleic Acids Res 40:8072-8084

14. Guinier A (1939) La diffraction des rayons $x$ aux tres petits angles: application a l'etude de phenomenes ultramicroscopiques. Ann Phys 12:161-237

15. Svergun DI (1992) Determination of the regularization parameter in indirect-transform 
methods using perceptual criteria. J Appl Cryst 25:495-503

16. Petoukhov MV, Konarev PV, Kikhney AG et al (2007) ATSAS 2.1 - towards automated and web-supported small-angle scattering data analysis. J Appl Cryst 40:s223-s228

17. Porod G (1982) General theory. In: Kratky O, Glatter O (eds) Small angle X-ray scattering. Academic Press, London, pp 17-51

18. Svergun DI (1999) Restoring low resolution structure of biological macromolecules from solution scattering using simulated annealing. Biophys J 76:2879-2886

19. Franke D, Svergun DI (2009) DAMMIF, a program for rapid ab-initio shape determination in small-angle scattering. J Appl Cryst $42: 342-346$
20. Konarev PV, Volkov VV, Sokolova AV et al (2003) PRIMUS: a Windows PC-based system for small-angle scattering data analysis. J Appl Cryst 36:1277-1282

21. Xu X, Reinle W, Hannemann F et al (2008) Dynamics in a pure encounter complex of two proteins studied by solution scattering and paramagnetic NMR spectroscopy. J Am Chem Soc 130:6395-6403

22. Funari E, Cavalieri M, Ade P et al (2000) Environmental and health problems of cyanobacteria blooms in surface waters in reference to the Italian situation. Ann Ig 12:381-400

23. Round AR, Franke D, Moritz S et al (2008) Automated sample-changing robot for solution scattering experiments at the EMBL Hamburg SAXS station X33. J Appl Cryst 41:913-917 